wirrung, störung (veranlaßt durch den aufenthalt und das hindernis von seite Tristans)', wo doch aber wohl der artikel nicht gut fehlen dürfte; viel besser wird der sinn durch 'in der ferne'.

16275 ze siner heimlîche er gewan von Gâles einen spilman. Bechstein läßt dem leser die wahl, ob er heimlîche als 'vertraulichkeit, freundschaft' oder als 'geheimnis' fassen will; auch Golther erklärt 'geheimnis, vertrauen'. Da nun aber heimlîche an mehreren stellen des Tristan (10415. 10595. 14301) 'privatgemach' bedeutet, ist mir am wahrscheinlichsten, daß es auch hier diesen sinn hat.

JENA.

ALBERT LEITZMANN.

\title{
ZU GUNDACKER VON JUDENBURG.
}

Gundackers gedicht 'Christi hort', von dem zuerst Pfeiffer in seinem Altdeutschen übungsbuch längere proben gegeben hatte, liegt uns nun in einem abdruck der einzigen handschrift (denn die ganz freie verarbeitung Zs. fda. 50, 386 hätte Schröder eigentlich gar nicht als fragment Gundackers bezeichnen dürfen) vollständig vor, den Jaksche, ein schüler von Kraus, für die texte der Berliner akademie besorgt hat (Berlin 1910). Man wird das nirgends bedeutende, aber in seiner naiven schlichtheit nicht ungefällige werk des Österreichers mit freude lesen, wenn auch stil und ausdruck keine reize haben, die literargeschichtlich fesseln könnten, mit einer ausnahme. Der ritterlich-höfische stil liegt Gundacker sicherlich so fern wie nur irgend etwas (das zeigt schon der gebrauch von punieren 5079 im sinne eines einfachen einherreitens), trotzdem aber hat die ausdrucksweise unsres eigenartigsten erzählers, Wolframs, ein paar vereinzelte strahlen, aus dritter oder vierter hand reflectiert, wie man wohl annehmen muß, bis in diese geistliche sphäre hineingesandt, die beachtet werden müssen. Ich setze, was ich aus Gundacker citiere, ins normale mhd. um. mich stichet rehte als ein dorn $566=$ 
ein more in stichet als ein dorn Parz. 66, 1; in (mit) jâmers siten 1573. $4168=\hat{u} z$ (mit, ze) jâmers siten Parz. 250, 1. 795, 1. Willeh. 8, 17. 73, 28. 467, 18; durch jâmers vurt 2090 $=$ in riuwen vurt Parz. 114, 4; an rehter vuore niht betrogen $3180=$ gein werder vuore niht betrogen Parz. 348, 12 (an küneclîcher vuore betrogen 118,2); über steine und über ronen muoz ich ungevertes wonen $3903=$ vil ungevertes reit er dan über ronen und über manegen stein Parz. 282, 6; dá von die helleclîchen (so hat Roethe das helfechlichen der handschrift zweifellos richtig gebessert) vart vuoren alle ir nâchkomen $4686=$ dar umbe die helleclîchen vart Adâmes geslehte vuor iedoch Willeh. 218, 16; liehtgemâl $4818=$ Parz. 64, 29. 144, 19. 243, 3. 263, 13. 565, 10. 619, 9. 661, 14. 694, 24. 695, 8. 706, 18. 717, 30. 723, 23. 727, 20. 730, 25. 732, 2. 740,20. 742, 28. 754, 16. 762, 17. 764, 20. 801, 3. 814, 12. Willeh. 16,5 . Tit. 7, 4. 43, 4 . $\mathrm{Zu}$ diesen Wolframischen reminiscenzen gesellen sich, damit auch ein reflex des größten lyrikers der klassischen zeit nicht fehle, zwei anklänge an Walther: swas kriuchet, vliuzet, vliuget oder bein ze der erde biuget $661=$ swaz kriuchet unde vliuget und bein zer erde biuget 8, 32; erzünde min herze schier mit dînem minneviure $1018=$ si sende uns got ze stiure bî sînem minneviure $6,18$.

Eine reihe von stellen in Gundackers gedicht geben mir zu bemerkungen veranlassung, die ich um so weniger zurückhalte, da Junk in seiner recension (Anz. fda. 35, 140) es gänzlich verabsäumt hat, auf Jaksches text und die erklärungen seines wortverzeichnisses im einzelnen einzugehen, man also leicht zu der ansicht verführt werden könnte, als sei hier alles in bester ordnung.

140 durch den val verriet er (Lucifer) Êven und Adâmen, daz si die gift nâmen: sîn val unser val geriet. Kraus will unser in unsern verändern und Jaksche erklärt daraufhin (s. 87) gerâten, wenn auch mit zweifelndem fragezeichen, als 'herbeiführen'. Ich glaube, es ist alles richtig überliefert, und möchte verstehen: 'sein fall wurde unser fall'. Dann läge hier ein beleg für die bedeutung von gerâten vor, die in Grimms wörterbuch 4, 1, 3573 mit dem nominativ aus dem Amadis belegt ist, während die gewobnliche construction die mit ze ist. 
308 der heilige geist kumt $\hat{u} f$ von dir im englischen gruß kann nicht richtig sein, aber Kraus' besserung nimt won in dir liegt graphisch $\mathrm{zu}$ weit $\mathrm{ab}$ und won =wonunge ist nicht einwandfrei belegt (vgl. Lexer 3,975). ' Steckt in $\hat{u} f$ von vielleicht iffen (vgl. Mhd. wb. 3, 178 a.) und in chumt etwa ruovet?

468 lese ich im engsten anschluß an die überlieferung daz des wîssagen vourde erfult und fasse wîssagen nicht als genetiv von wîssage, was allerdings nötigen würde, mit Jaksche wort zu ergänzen und dadurch den vers zu überladen, sondern als stn. im sinne von 'weissagung' (vgl. Maria 176, 22; Judith Diemer 148, 6; Laub. Barl. 1304).

696 und 698 lese ich dîn vïeze statt die vïeze.

931 einer sun der muoter sîn: einer im sinne von 'einziger', wofür Kraus einec lesen will, könnte vielleicht trotz des mangels paralleler belege richtig sein.

992. Jaksche verewigt einen schreibfehler der handschrift, wenn er (s. 89) ein swm. toere statt tôre ansetzt.

1097. Unter mandât bemerkt der herausgeber im wortverzeichnis (s. 88), die stelle zeige deutlich die bedeutung 'mahlzeit, abendmahl', und die von den wörterbüchern angesetzte bedeutung 'fußwaschung' scheine ihm für alle dort angeführten belege nicht zu stimmen. Der ausdruck stammt aus dem kirchlichen ritus, wo am gründonnerstag; wenn nach absingung des evangeliums die fußwaschung beginnt, der chor die antiphon 'Mandatum novum do vobis, ut diligatis invicem, sicut dilexi vos, dicit dominus' (Joh. 13, 34) singt, und bedeutet ursprünglich sicherlich nichts andres als 'fußwaschung': vgl. Otfr. 4, 11, 11 er stuant yr themo muase thô $z i$ themo âbande, legita sîn giwâti, er in mandât dâti und Gen. 31, 44 dô er (Abraham) iz (das kalb) ersluog, die vuoze er in (den engeln) dwuog; bedaz er getete die mandâte, sô was daz ezzen gesoten jouch gebrâten. Wenn man sich erinnert, daß die fußwaschungsscene nach der Johanneischen darstellung ein mittlerer teil des letzten abendmahls ist ('surgit a coena' 13,4 ; 'cum recubuisset iterum' 12), so liegt auf der hand, daß die bedeutung des wortes mandâte leicht in diesem sinne erweitert werden konnte, daß man 'letztes abendmahl einschließlich fußwaschung' darunter verstand. So bedeutet denn Gundackers mandâten 1106 
zweifellos 'mahlzeit halten'. Aber ein zweifel an der richtigkeit der bedeutung, die unsre wörterbücher angeben, ist dadurch nicht im mindesten gerechtfertigt und nur an ganz wenigen stellen außer Gundacker ist die erwähnte erweiterung der bedeutung belegbar.

1215 daz dîn süezer lîp klâr mit bluote was zeflozzen gar: es ist wohl bevlozzen zu lesen.

Die fehlende zeile 1250 ist wohl der der verrâtcere was oder ähnlich zu ergänzen.

1402 er hât sich des îz getân, er sî kïnec. Jaksche erklärt (s. 90) sich $\hat{u} z$ tıon durch 'sich für etwas ausgeben': richtiger wäre 'sich einer sache anmaßen'.

1502 ôwê heia und hei: ich lese ôwê unde heiâ hei, wie Parz. 103, 20. 160, 3. 407, 16. 496, 22 und sonst sich findet.

1762. Roethes veränderung von die in hie, wodurch ein verbotener rührender reim entsteht, kann ich nicht billigen: dieser reim scheint mir härter als das enjambement, das die überlieferung bietet und das er beseitigen soll.

1800 des lasters nam Pilâtus phlilt. Das heißt nicht, wie Jaksche (s. 89) erklärt, 'er nahm anstoß', sondern nur 'anteil', was in diesem speciellen falle allerdings negativer, miBbilligender anteil ist. So hat der herausgeber noch mehrfach übersetzungen im wortverzeichnis gegeben, die zwar für die einzelne stelle passen mögen, aber in sich nicht genau, ja fehlerhaft sind: so setzt er für unwende (s. 90) 'sicher' statt 'unabwendbar, nicht zu ändern' als bedeutung an und erfindet sogar (ebenda) ein ursprinc im sinne von 'urheber, erreger', weil das wort sich an den beiden stellen auf eine lebendige person bezieht.

2018. Das überlieferte bidwen war zu halten und nicht mit Kraus in bidmen zu ändern, da es sich auch Fundgr. 1, 196, 35 findet.

2056. Viele belege für das masc. passiôn stehen im Dwb. 7, 1489, wo noch nachzutragen ist: Alex. A 6i6; Fastn. 329, 14. 679, 9; Folz, Meisterl. s. 8; Schade, Sat. u. pasqu. 2, 57. 58.

2618 steckt in dem handschriftlichen an einer doch wohl wan ein, also: dû bist wan cin pilcgrim. 
2874 möchte ich lesen: saget uns, wes kaphet ir hin an sô tiure Jêsî Krist hin nâch? 'warum gafft ihr so eifrig hinauf hinter Jesus Christus her?' Dann kann das überlieferte Jesu, das Kraus geändert hat, erhalten bleiben.

2955 die komen Rômoere als ïbersetzung von 'advenae Romani' (Acta apost.. 2,.10) -ist ganz in der ordnung, wenn man komen als plur.. des particips faßt, und Kraus' änderung von die in zuo erübrigt sich dann.

2996 daz predigten si vil genôte' 'das predigten sie sehr eifrig'. Ein arger schnitzer ist es, wenn Jaksche, das geläufige adverb genôte als verbalform mißverstehend, ansetzt (s. 87): 'genceten, einen, stark beschäftigen, in anspruch nehmen', wozu er selber bereits ein zweifelndes fragezeichen setzt.

3258 als man liebe vriunt sol: liest man lieben, so entsteht wörtliche übereinstimmung mit 4338 .

4342 er schist von danne âne allen haz und vuor zehant vrôlîch: ich lese ze lande statt zehant.

4658 sînes tôdes nie mensche sô leide wurt. Warum Roethe das überlieferte laid in laidic ändern will, sehe ich nicht ein, da gar kein anstoß in dem überlieferten ist: vgl. Ath. $A^{*} 9$.

4765 im ebengewaltec und hêr: mit 2991 lese ich ebenhêr.

5225. In Kraus' besserung ist das ir zu streichen, denn under krône ohne artikel ist die gewöhnliche wendung: vgl. Nib. 595, 4. 631, 3. 659, 2. 755, 3. 1314, 4. 1616, 4. 1708, 4; Willeh. 462, 2; Tit. 146, 2; Ernst B 5912.

Mit vers 5294 schließt Gundackers dichtung ab. Was in der handschrift und in Jaksches ausgabe noch folgt; die verse 5295-5320 mit der überschrift: 'Von gote ein guot gebet', hat nichts damit $\mathrm{zu}$ tun, sondern ist, was sowohl Jaksche als Roethe und Kraus entgangen ist, wörtlich das erste der beiden gebete, mit denen Freidank seine Bescheidenheit abschließt $(180,8-181,9)$, und nur vom schreiber als epilog angefügt.

JENA.

ALBERT LEITZMANN. 